\title{
Index of Manuscripts
}

Aachen, Domschatzkammer, Treasury Gospels 104

Aachen, Domschatzkammer, Liuthar-Gospels 210

Aberdeen, University Library, Papyrus 2a (Gospel of John, fragmentary) 58

Addis Ababa, National Library, MS 28 (KTN) 72, 75-77, 79, 85, 86, 90

Addis Ababa, The Institute of Ethiopian Studies, 3475a-b

(fragmentary) (IES-3475) 70, 72, 75, 76, 79, 85, 90

'Adwa, Abba Garima Monastery, Abba Garima Gospels I (AG I) X, 29, $67,72,73,74,75,76,79,81,83,85,87,88,90,99,104-105$, 109-111, 123, 125, 135, 210

'Adwa, Abba Garima Monastery, Abba Garima Gospels II (AG-II) X, 29, 67, 75, 99, 104, 105, 109-111, 127, 210

'Adwa, Abba Garima Monastery, Abba Garima Gospels III (AG-III) X, 29, 67, 71-73, 81, 83, 85, 88, 92, 99, 104, 105, 106, 107, 109-111, 120, 121, 122, 127, 141, 197, 198, 210

Akkälä Guzay, Däbrä Libanos (DLB) 72, 73, 75, 79, 81, 83, 85, 90

Alba Julia, Biblioteca Documentară Batthyáneum, MS R II 1 (Lorsch Gospel, part I) 147, 194, 195

Amba Dära, Maryam Mägdälawit (MMG) 72, 73, 79, 82, 85, 86

Angers, Bibliothèque municipale, MS 24142

Athens, National Library of Greece, Cod. 92 (GA 1410) 34

Augsburg, Universitätsbibliothek, Oettingen-Wallersteinsche Bibliothek, Cod.I.2.4.2 (Gospels from Echternach / Augsburg Gospels) 58

Autun, Bibliothèque Municipale, Cod. 3 (Gundohinus Gospels) 181

Autun, Bibliothèque Municipale, Cod. 4198

Avranches, Bibliothèque Municipale, 48, 66, 71 (Gospel Book Fragments) 60

Baltimore, Walters Art Museum, MS W.4 178

Baltimore, Walters Art Museum, MS W.26 (Claricia Psalter) 209, 232, 236, 237

Baltimore, The Walters Art Museum, MS W.836 (WAM-836) 72, 75, $76,78,79,81,83-86,88$

Bamberg, Staatsbibliothek, Patr. 86 (B.V.13) 174

Berlin, Staatsbibliothek zu Berlin - Preußischer Kulturbesitz, Phillips 1815 (Cod. Berolinensis Phillippicus) 101

Berlin, Staatsbibliothek zu Berlin - Preußischer Kulturbesitz, Ms. lat. fol. 416 (Vergilius Augusteus) 11

Berlin, Staatsbibliothek zu Berlin - Preußischer Kulturbesitz, Ms. or. quart. 102087

Berlin, Staatsbibliothek zu Berlin - Preußischer Kulturbesitz, Ms. theol. lat. fol. 733 (Prüm Gospels) 147, 149, 167

Berlin, Staatsbibliothek zu Berlin - Preußischer Kulturbesitz, Ms. theol. lat. fol. 485 (Quedlinburg Itala) 140

Bern, Burgerbibliothek Bern, Cod. 212101

Brescia, Biblioteca civica Queriniana, s.n., Manoscritto Purpureo (Codex Brixianus) 42, 177, 178

Brescia, Biblioteca civica Queriniana, Cod. G VI.7 (Memoriale of Brescia) 224

Boulogne-sur-Mer, Bibliothèque municipale, MS 8 146, 147

Brussels, Bibliothèque des Bollandistes, MS 299181

Brussels, Bibliothèque Royale, MS 2034-35 (Sacramentary of Abbot Wibald) 246

Cambridge, Corpus Christi College, Parker Library, MS 286 (St Augustine Gospels) 103, 107, 112, 133-144, 146, 147, 167, 170
Cambridge, Fitzwilliam Museum, MS 45-1980 145-147

Cambridge, Magdalene College, Pepysian 2981 (I) 174

Cambridge, Trinity College, MS B.10.4 155, 156-166, 247

Cambridge, Trinity College, MS R.17.1 (Canterbury Eadwine Psalter) 139

Chantilly, Musée Condé, no. 1565414

Cividale del Friuli, Museo Archeologico Nazionale, MS CXXXVII (Elisabeth Psalter) 229

Cologne, Erzbischöfliche Diözesan- und Dombibliothek, MS 2247

Cologne, Erzbischöfliche Diözesan- und Dombibliothek, MS 12 (Hillinus-Codex) 104

Cologne, Erzbischöfliche Diözesan- und Dombibliothek, Cod. $14 \quad 210-215$

Cologne, Erzbischöfliche Diözesan- und Dombibliothek, Cod. 1001a (St. Maria ad Gradus Gospels) 211, 216-219

Cologne, Erzbischöfliche Diözesan- und Dombibliothek, Cod. 212224

Darmstadt, Hessisches Landesmuseum, Kg 54:211/ AE 680247

Däqqi Dašəm, Qəddus Mika'el (DDM) 72

Diyarbakir, Meryem Ana Kilisesi, Hill Museum and Manuscript Library, no. diyr 339 (Diyarbakir Gospels) 88, 107, 123

Dublin, Royal Irish Academy, MS D.II.3 (Stowe St John) 48

Dublin, Trinity College Library, MS 52 (Book of Armagh New Testament) 47-49, 53, 56, 58

Dublin, Trinity College Library, MS 55 (Codex Usserianus Primus) 48, 181

Dublin, Trinity College Library, MS 57 (Book of Durrow) 12, 48, 49, 51, $56,58,64,87$

Dublin, Trinity College Library, MS 58 (Book of Kells) 12, 48-50, 58, $64,87,141,150,166,173$

Dublin, Trinity College Library, MS 59 (Book of Dimma) 48

Dublin, Trinity College Library, MS 60 (Book of Mulling) 47-49, 54, $56,58-60,63,64$

Durham, Cathedral Library, MS A. II. 10 (Durham Gospel Book, fragmentary) 60

Durham, Cathedral Library, MS B.iv.9 (Prudentius, Works) 102

Escorial, Library of the Monastery of San Lorenzo, Cod. Vitrinas 17 (Speyer Gospels, Codex aureus Escorealensis) 14

Essen, Münsterschatz, MS 1 (Essen Gospels) 178

Florence, Biblioteca Medicea Laurenziana, Orient. 81 (Tatian, Diatessaron) 12, 13, 81

Florence, Biblioteca Medicea Laurenziana, Amiatino 1 (Codex Amiatinus) 56, 105, 107, 108, 117

Florence, Biblioteca Medicea Laurenziana, Plut. 1.56 (Rabbula Gospels) $76,87,88,90,103-105,107,110,116,140,143,155$, 181

Fulda, Landesbibliothek, Cod. Bonifatianus 1 (Codex Fuldensis) 17, 21-26, 29, 174, 177

Fulda, Landesbibliothek, Cod. Bonifatianus 3 (Cadmug Gospels) 47, 48

Glasgow, University of Glasgow Library, MS Hunter 475 (GA 560) 35 Gär'alta, Däbrä Mä'ar (DMR) 69, 70, 72, 73, 76, 79, 81, 83, 85, 86, 90 Goslar, Stadtarchiv, Hs. B 4387 (Goslar Gospels) 247 Gulo Mäkäda, 'Ura Mäsqäl (UML) 72, 73, 76, 79, 81, 83, 85, 86, 90 
Ḥayq, Däbrä Ḥayq $\exists$ sțifanos (IMA) $72,73,76,79,85,86,90$

Istanbul, Türk ve İslam Eserleri Müzesi, ŞE 321106

Ivrea, Biblioteca Capitolare, Cod. Eporediensis LXX 101

Jerusalem, Armenian Patriarchate, MS 2555 (Second Etchmiadzin

Gospels) 81, 83, 86

Lake Ṭana, Däq, Arsima Säma' ətat (AST) $72,73,76,79,83,85,88$ Lake Ṭana, Kəbran, Kəbran Gäbrə'el (KGL) 72, 75, 76, 79, 83, 85, 86 Laon, Bibliothèque municipale, MS 63 147, 148

Leiden, Bibliotheek der Rijksuniversiteit, Voss. Lat. F. 26174

Leningrad, see St Petersburg

London, British Library, Add. 5111/1 (Golden Canon Tables) 33, 86, 141,155

London, British Library, Add. 5463135

London, British Library, Add. 12134105

London, British Library, Add. 14429105

London, British Library, Add. 14445 35, 105, 118

London, British Library, Add. 17739 (Jumièges Gospels) 247

London, British Library, Add. 37472139

London, British Library, Add. 40618 (Irish Gospels) 48

London, British Library, Add. 43725 (Codex Sinaiticus [GA 01]) 30

London, British Library, Add. 49598 (Aethelwold

Benedictional) 209, 210, 225, 228, 229, 233, 246

London, British Library, Cotton MS Domitian A VII (Liber Vitae of Durham) 211

London, British Library, Cotton MS Galba A XVIII (Galba Psalter) 225, 229, 233

London, British Library, Cotton MS Nero D IV (Lindisfarne Gospels) 12, 33, 49, 56

London, British Library, Harley MS 647 (Cicero, Aratea) 101

London, British Library, Harley MS 177587

London, British Library, Harley MS 2788 (Harley Golden Gospels) 87, 90, 138, 150, 151, 166, 181, 187, 201, 202, 205, 206

London, British Library, Or. 69186

London, British Library, Royal MS 1 D VIII (Codex Alexandrinus [GA 02]) 30

London, British Library, Royal MS I E VI (Gospel book, the 'Royal Bible') 58, 64, 135

London, Lambeth Palace Library, MS 1370 (MacDurnan Gospels) 47-49, 55, 56, 58, 63, 64

Los Angeles, Getty Collection, MS 64 (Stammheim Missal) 209, 210, 229, 230-232, 234-235, 247

Manchester, John Rylands Library, MS lat. 10104

Maseyck, Church of St Catherine, Codex A and B 87

Mount Athos, Stauronikita Monastery 76

Mestia, History and Ethnology Museum of Svanetia, Adysh Gospels 75, 81

Munich, Bayerische Staatsbibliothek, Clm 4454 (Gospels of Emperor Henry II) 205

Münster, Landesarchiv Nordrhein-Westfalen, Abteilung Westfalen, Msc. I 133 (Liber Vitae from Corvey) 210, 242-247

New Haven, Yale University Library, P. Dura 10. 0212 (Dura Parchment) 26

New York, The Metropolitan Museum of Art, acc. no. 1998.66 (MET-1998.66) 72, 75, 76, 79, 85, 86
New York, Metropolitan Museum of Art, acc. no. 2006.100 (Ethiopic Gospels, second fragment)

$5,6,67-70,72,73,75,76,79,81,83,85,86,88-90,92$

New York, Metropolitan Museum of Art, acc. no. 2015.560 (Gospels of Queen Theutberga) 181

New York, Metropolitan Museum of Art, X.455 (LDAB 1062) (Epiphanius canons, fragmentary) 42, 141, 178

New York, The Morgan Library \& Museum, M.828 (ZGL) (Gospels of Zir Ganela) 72, 73, 76, 85, 141

New York, The Morgan Library \& Museum, M.869 (Arenberg Gospels) 166, 247

Orléans, Bibliothèque municipale, MS 184 (Fleury Prayer Book) 63 Oxford, Bodleian Library, MS Auct. F. 2.13 (Terence's Comedies) 104 Oxford, Bodleian Library, MS Douce 293140

Paris, Bibliothèque de l'Arsenal, MS 1171 (Sainte-Aure Gospels) 144 Paris, Bibliothèque nationale de France, MS arabe 324C 106 Paris, Bibliothèque nationale de France, MS arabe 330c 106 Paris, Bibliothèque nationale de France, MS éthiopien 32

(BNF-32) 70, 72, 73, 76, 79, 83, 85, 86

Paris, Bibliothèque nationale de France, MS grec 64 (GA 15) 33

Paris, Bibliothèque nationale de France, MS grec 79 38-44

Paris, Bibliothèque nationale de France, MS grec 91 (GA 10) 30, 31, 33, 34

Paris, Bibliothèque nationale de France, MS grec 177 (GA 299) 34, 36,37

Paris, Bibliothèque nationale de France, MS grec 230 (GA 12) 32, 33, 35

Paris, Bibliothèque nationale de France, MS lat. 116 (St Denis Bible) 247

Paris, Bibliothèque nationale de France, MS lat. 257142

Paris Bibliothèque nationale de France, MS lat. 281, MS lat 298 (Codex Bigotianus) 60, 61

Paris, Bibliothèque nationale de France, MS lat. 2421102

Paris, Bibliothèque nationale de France, MS lat. 7899 (Terence Parisinus) 104,114

Paris, Bibliothèque nationale de France, MS lat. 8850 (Soissons Gospels) 88, 89, 138, 147, 150-155, 166, 167, 193, 195-197, 202, 203, 206, 246-247

Paris, Bibliothèque nationale de France, MS lat. 9389 (Echternach Gospels / Gospels of St Willibrord) 12, 48, 49, 178

Paris, Bibliothèque nationale de France, MS lat. 11553 (Codex Sangermanensis primus, Bible) 60

Paris, Bibliothèque nationale de France, MS lat. 13169 (Codex Sangermanensis secundus, Gospel Book) 60

Paris, Bibliothèque nationale de France, MS lat. 11627224

Paris, Bibliothèque nationale de France, MS lat. 17225177

Paris, Bibliothèque nationale de France, MS lat. 17968104 Paris, Bibliothèque nationale de France, MS nouv. acq. lat. 1203

(Godescalc Lectionary) 81, 83, 150, 167, 193, 195, 197

Paris, Bibliothèque nationale de France, MS nouv. acq. lat.1587 (St Gatien Gospel) 60,62

Paris, Bibliothèque nationale de France, MS nouv. acq. lat. 2334 (Ashburnham Pentateuch) 105, 107, 108, 117

Paris, Bibliothèque nationale de France, MS suppl. gr. 1286143

Paris, Bibliothèque nationale de France, MS syr. 97105

Paris, Bibliothèque nationale de France, MS syr. 341 (Peshitta Old and New Testament) 90, 103, 107, 109, 113, 122 
Piacenza, Biblioteca Comunale Passerini-Landi, s.n. (Angilberga Psalter) 187

Poitiers, Médiathèque François-Mitterrand, MS 17 (65), Réserve précieuse (Sainte Croix of Poitiers Gospels) 58, 166, 173-190

Princeton, Princeton University Library, cod. Garrett 676

Private Collection (Ethiopic Gospel Book) (PC-3) 72, 79, 85

Rossano, Museo Diocesano e del Codex, s.n. (Rossano Gospels / Codex Purpureus Rossanensis) 103-105, 107, 143

Salzburg, Erzabtei St. Peter, Archiv, Hs. A 1 (Confraternity book of St Peter's Salzburg) 224

Șan'ā', Dār al-Makhtūtāt, DAM 20-33.1 (Umayyad Qur'ān of Șan'ā') 106, 118

Säraye, Däbrä Maryam Qwäḥayn (DMQ) 72, 73, 76, 83, 85, 86

Șəra', Däbrä Șärabi (DSB) 72, 76, 79, 80, 83, 85, 86, 90

St Gall, Stiftsarchiv, Cod. C 3 B 55 (Confraternity book of St Gall) 224

St Gall, Stiftsarchiv, Cod. Fab. 1 (Liber viventium Fabariensis / Liber viventium of Pfäfers) 209, 210, 211, 220-225, 229, 246

St Gall, Stiftsbibliothek, Cod. Sang. 23 (Folchard Psalter) 209, 210, 225-228, 233, 246

St Gall, Stiftsbibliothek, Cod. Sang. 50178

St Gall, Stiftsbibliothek, Cod. Sang. 51 (Irish Gospel) 48

St Gall, Stiftsbibliothek, Cod. Sang. 60 (Gospel of John) 56

St Gall, Stiftsbibliothek, Cod. Sang. 232 (Isidore of Seville, Etymologiae) 200

St Gall, Stiftsbibliothek, Cod. Sang. 1395 (Veterum Fragmentorum Manuscriptis Codicibus detractorum collectio) 58

St Petersburg, National Library of Russia, Cod. Marcel 13 106, 109, 119

St Petersburg, National Library of Russia, Lat. F.v. I.8 (Leningrad Gospels) 60

St Petersburg, National Library of Russia, Lat. O.v.I.1. (Gospel Book Fragments) 60

St Petersburg, National Library of Russia, Hebrew II B 49106

Stockholm, National Library of Sweden (Royal Library), A. 135 (Codex Aureus) 12, 193

Stockholm, Stockholm National Museum, MS B 196115

Stockholm, Stockholm National Museum, MS B 2034 (Ethiopic Gospels, first fragment) (NMB-2034) 5, 6, 69, 70, 86

Stuttgart, Württembergische Landesbibliothek, HB I 240 (Liber Litaniarum et Benedictionum) 228

Stuttgart, Württembergische Landesbibliothek, HB II 24 (Landgrave Psalter) 209, 232, 233, 238-241, 246

Täkwəlädäre, Boru Meda Abärra Śəllase (BSS) $72,73,76,79,83$, 85,86

Trèves, Séminaire, MS 40177

Trier, Trierer Domschatz, MS 61225

Trier, Stadtbibliothek, cod. 22 (Ada Gospels) 173

Trier, Stadtbibliothek, cod. 171/1626 14

Troyes, Bibliothèque municipale, MS 138177

Uppsala, University Library, MS C 93 (Codex Caesareus) 11, 14 Utrecht, University Library, MS 3285

Vatican City, Biblioteca Apostolica Vaticana, MS Arch. S. Pietro H. 19 (Terentius Basilicanus) 104

Vatican City, Biblioteca Apostolica Vaticana, MS Barb. lat. 2154 (The Codex Calendar of 354) 87, 104, 115, 229
Vatican City, Biblioteca Apostolica Vaticana, MS Pal. lat. 47 (SaintBertin gospel book) 144, 146

Vatican City, Biblioteca Apostolica Vaticana, MS Pal. lat. 50 (Lorsch Gospels, part II) 147, 181

Vatican City, Biblioteca Apostolica Vaticana, MS Pal. lat. 1564 (Codex Agrimensorum) 106, 119

Vatican City, Biblioteca Apostolica Vaticana, MS Pal. lat. 1713 (Optatianus Porfyrius) 101, 102

Vatican City, Biblioteca Apostolica Vaticana, MS Reg. lat. 733102 Vatican City, Biblioteca Apostolica Vaticana, MS Vat. gr. 33487 Vatican City, Biblioteca Apostolica Vaticana, MS Vat. gr. 75189 Vatican City, Biblioteca Apostolica Vaticana, MS Vat. gr. 1291 (Vatican Ptolemy) 101, 104

Vatican City, Biblioteca Apostolica Vaticana, MS Vat. gr. 161390

Vatican City, Biblioteca Apostolica Vaticana, MS Vat. lat. 3225 (Vergilius Vaticanus) 103, 108, 141

Vatican City, Biblioteca Apostolica Vaticana, MS Vat. lat. 3256 (Vergilius Augusteus) 11

Vatican City, Biblioteca Apostolica Vaticana, MS Vat. lat. 3806 14, 135

Vatican City, Biblioteca Apostolica Vaticana, MS Vat. lat. 3867 (Vergilius Romanus) 103, 107, 112, 122

Vatican City, Biblioteca Apostolica Vaticana, MS Vat. lat. 3868 (Vatican Terence) 103, 106, 107, 113, 114

Vatican City, Biblioteca Apostolica Vaticana, MS Vat. lat. 546587

Vatican City, Biblioteca Apostolica Vaticana, MS Vat. lat. 5729155

Vendôme, Bibliothèque municipale, MS 0002177

Venice, Abbey of San Lazzaro degli Armeni, Biblioteca, MS $1144 / 8687$

Venice, Biblioteca Nazionale Marciana, MS Gr. 1883

Verona, Biblioteca Capitolare, Cod. VI 48, 60

Verona, Biblioteca Capitolare, Cod. LX (58) 87

Vienna, Österreichische Nationalbibliothek, Cod. 847 88, 104

Vienna, Österreichische Nationalbibliothek, Cod. 940 49, 52, 56, $58,63,64$

Vienna, Österreichische Nationalbibliothek, Cod. 997 56, 57

Vienna, Österreichische Nationalbibliothek, Cod. 1224 (Cutbercht Gospels) 206

Vienna, Österreichische Nationalbibliothek, Cod. Medicus Graecus 1 (Vienna Dioscorides) 106, 107, 120

Vienna, Österreichische Nationalbibliothek, Cod. Theol. gr. 15487

Vienna, Österreichische Nationalbibliothek, Cod. Suppl. gr. 52 (GA 3) 34,35

Wolfenbüttel, Herzog-August-Bibliothek, Cod. Guelf. 36.23 Aug. $2^{\circ}$ (Codex Arcerianus) 104, 107, 115

Wolfenbüttel, Herzog August Bibliothek, Cod. Guelf. 105 Noviss. $2^{\circ}$ (Gospels of Henry the Lion) 247

Wolfenbüttel, Herzog-August-Bibliothek, Cod. Guelf. 99 Weiss. (Augustinian sermons) 224

Würzburg, Universitätsbibliothek, M.p.th.f.68 (Burchard Gospels) 63

Yerevan, Matenadaran, Mesrop Mashtots Institute of Ancient Manuscripts, MS 2374 (formerly Etchmiadzin MS 229) (Etchmiadzin Gospels) 83, 103, 124, 204

Yerevan, Matenadaran, Mesrop Mashtots Institute of Ancient Manuscripts, MS 943075

Zurich, Zentralbibliothek, Rh. hist. 27 (Reichenau confraternity book) 224 


\section{Index of Names}

Aaron 113, 143

Abraham 205

Achilles Tatius 102

Adalbert of Corvey, provost 246

Adalhard of Corbie 190

Adelricus 103

Aethelwold of Winchester, bishop 225

Agnes of Poitou 10, 11

Afra, saint 232

Agennius Urbicus 104, 115

Ailerán of Clonard 102, 187

- Poem Canon evangeliorum 58, 186, 187, 189

Albertus Magnus 201

Alexander the Great 101

Allen, Garrick 33

Alcuin of York 63, 187

Ambrose of Milan 138

'Amdä Șəyon I, emperor 70

Ammonius of Alexandria VII, 18, 19, 21-23, 35, 42, 76, 140, 177

- see Eusebius of Caesarea, Letter to Carpianus, Ammonius quidem

Andrew II, king of Hungary 233

Anicia Juliana 106

Andrist, Patrick 30

Antoninus Pius, emperor 209

Aratus of Soli 101

Arno of Salzburg, bishop 63

Augustine of Hippo 25, 29, 34, 133, 199, 201, 233

Ausonius 101

Balicka-Witakowska, Ewa 70

Bandmann, Günter 209

Barber, Charles 142

Barbet-Massin, Dominique 63

Bausi, Alessandro 47, 67, 75, 89, 99

Bede 138

Benedict Biscop 143

Bernard, Émile 4

Bernward of Hildesheim, bishop 229

Berthold of Weingarten, abbot 228

Bischoff, Bernhard 56, 63

Bjuström, Per 1, 8

Boeckler, Albert 2,14

Borst, Arno 201

Bracciolini, Poggio 199

Bruno, Giordano 199

Buchthal, Hugo 2

Budny, Mildred 139, 140, 142, 171

Carpianus VII, 13

- see Eusebius, Letter to Carpianus (Ammonius quidem)

Cassiodorus, Flavius Magnus Aurelius 21

Cebes 102

Charlemagne 190,193, 195, 199, 204

Chartier, Roger 30, 44

Christ, Jesus 10, 20, 22-24, 26, 30, 44, 75, 76, 81, 88, 90, 102, 103, $107,108,123,134,138-141,143,147,150,155,164,166,167$,
$181,189,190,195,198,199,201,203,205,206,209,224,225$, 228, 232, 233, 246, 247

Christina, Queen of Sweden 8, 9

Cicero 199

Claussen, Peter Cornelius 204

Clemen, Paul 2

Constance, queen of Bohemia 233

Constantine I VII, 101, 195

Constantius II 104

Coogan, Jeremiah 17, 139

Cox, Catherine 47

Crawford, Matthew R. 63, 140

Damasus I, pope 22, 101, 209

Damoetas 112

Dawit II, emperor 72

David 155, 225

De Bruyne, Donatien 58, 174, 177

De Hamel, Christopher 170

Democritus 199

Der Nersessian, Sirarpie 103

Deshman, Robert 225

Devens, Monica S. 70

Diego, Benjamin 141

Dioscorides 106, 107, 120

Ealhburg 170

Einhard 201

Ellenius, Allan 1

Elsner, Jaś 141

Ephrem the Syrian 26

Eusebius of Caesarea 13, 18, 20-24, 26, 29, 33, 34, 42, 44, 56, 99 , 100-102, 133, 134, 138, 143, 155, 167, 174, 177, 198, 210

- Eusebian system / apparatus VII-IX, 17, 21, 23-25, 29, 30, 33-35, 42, 44, 45, 47, 49, 56, 58, 60, 63, 64, 67, 69, 70, 76, 79, $81,86-88,90,99,100,133-135,140,150,151,155,166,178$

- Ecclesiastical History 21-22

- Letter to Carpianus, Ammonius quidem VII, VIII, 6, 13, 18, 21, $34,42,44,67,69,88,90,100,101,104,105,106-110,120$, 124, 125, 155, 173-175, 177, 178, 210

- Portrait of Eusebius 106-108

Ezekiel, prophet 143

Ezra 107

Fiaccadori, Gianfranco 70

Ficino, Marsilio 199

Flavius Josephus 203

Fogg, Samuel 16

Fouquet, Jean 203

Friend, Albert Matthias Jr. 106

Galla Placidia 102

Gallus 104

Ganz, David 177

George, saint 233

Gervers, Michael 67

Gertrud, queen of Hungary 233 
Gibbon, Edward 18

Goldschmidt, Adolph 2

Goody, Jack 17, 19, 20, 26

Goya, Francisco 4

Grabar, André 2, 10

Gregory the Great 14, 135, 189

- Homilies on Ezekiel 189

Gregory, Caspar 34

Greenblatt, Stephen 199

Guidi, Ignazio 85

Habbakuk, prophet 107,122

Hackspill, Louis 85

Haggai, prophet 107

Hanaghan, Michael 21

Hartmut of St Gall, abbot 225

Haseloff, Arthur 2

Heldman, Marilyn E. 70

Henry II, emperor 205

Henry III, emperor 10, 11

Herbert, Lynley Anne 166, 232

Hermann I, landgrave of Thuringia 233

Herod, king of Judea 142

Hilduin of Saint-Denis, abbot 246

Houghton, Hugh 47, 63

Hrabanus Maurus 233

Irenaeus 133

Isaiah, prophet 155, 202

Isidore of Seville 21, 63, 193, 199-201

James, apostle 107

Jerome IX, 21, 56, 63, 101, 133, 173, 174, 178, 187, 198, 199, 209,

211, 218, 219, 247

- Commentary on Ezekiel 224

- Prefatory texts 150, 151, 166, 193

Novum Opus 22, 49, 56, 58, 63, 151, 174, 178-179

Plures fuisse 147, 150-151, 174, 178

- Vulgate translation 177

Jesse of Amiens 190

Job 103

Joel, prophet 107

John the Baptist 138, 142, 198, 233

John the Evangelist 24, 34, 107, 116, 123, 135, 138, 140, 145, 146, 147, 151, 166, 198, 211

Joseph, saint 147

Joseph of Arimathea 23-25

Jude, saint 10

Jülicher, Adolf 29, 30, 44

Kelber, Werner H. 17-20, 26

Koehler, Wilhelm 2

Kupfer, Marcia 133

Lazarus 140

Leal, Beatrice 89

Leatherbury, Sean 89

Leo III, pope 190

Lepage, Claude $70,86,89$

Leroy, Jules 67, 89, 90, 109

Leucippus 199
Levi 138, 139, 141

Longus 102

Lowden, John 81, 142, 143

Lowe, Elias Avery 11

Lucian 102

Lucretius, Titus 193, 199-201

Luke the Evangelist 24, 34, 58, 107, 112, 116, 121, 135, 137-139, 142, 144, 147, 151, 166, 167, 189, 211

MacNamara, Martin 47

Malraux, André 8

Mantegna, Andrea 198

Marcion 22

Mark the Evangelist $24,34,58,105,107,116,121,135,138,147,151$, 166, 189, 211

Mary, Virgin 138, 141, 147, 150, 228, 232, 233

Matthew the Evangelist 24, 34, 48, 107, 110, 116, 135, 138, 147, 166, 189, 195, 211

Matthew, apostle 76

Maurice, saint 233

McGurk, Patrick 47, 49, 58, 63, 174, 178

McKenzie, Judith X, 67, 99, 106, 110

McLaughlin, Anne 133, 171

Meehan, Bernard 48

Meingoz of Weingarten, abbot 228

Michah, prophet 107

Menalcas 112

Michael, archangel 232

Mills, Ian 26

Minard, Pierre 174, 177

Mo'a, lyäsus 72

Moses 113, 143, 201, 205

Mullins, Elizabeth 17

Nees, Lawrence 92,181

Nersēs Šnorhali 102, 204, 205

Netz, Reviel 20

Neugebauer, Otto 33

Nicolas, saint 232, 246

Nordenfalk, Carl VIII-X, 1-5, 8-14, 16, 17, 29, 47, 67, 104, 155, 173, 178, 181, 193, 195, 209, 210, 224, 225

Nordenfalk, Katarina 1

Obadiah, prophet 107

Ó hAnnracháin, Tadgh 47

Ó Cróinín, Dáibhí 47

O’Driscoll, Joshua 144

Oldjira, Meseret 141

O’Loughlin, Thomas 17, 56, 58

Olsen, Kåre 4

Ong, Walter 18

Optatian Porphyry 101

O'Reilly, Jennifer 48

O'Reilly, Terence 47

Origen of Alexandria VII, 18

Osterman, Gunhild 1

Otakar I, king of Bohemia 233

Otto II, emperor 14, 205

Ovid 199, 201 
Pächt, Otto 2

Palaemon 112

Panofsky, Erwin 3

Patrick, saint 49

Paul, apostle 22, 58, 155, 199, 206

- epistles 21, 22,73

Paul VI, pope 8

Penna, Angelo 34

Peter, apostle 138, 139, 155, 193, 203

Phoenix, Robert 16

Pilate, Pontius 30

Pliny the Elder 102, 193, 199, 201

Plumpe, Joseph C. 199

Poilpré, Anne-Orange 167

Porfirius, Publilius Optatianus 101

Ptolemy, Claudius 33, 101

Pyrrhus, king 199

Ranke, Eduard 21, 23, 25

Rembrandt van Rijn 5, 7, 8

Reudenbach, Bruno 47, 99, 133, 210

Riggsby, Andrew 20, 25

Roantree, Dermot 47

Rolfsen, Alf 16

Rosenthal, Jane 166

Rosenwald, Lessing J. 8

Rufinus of Aquileia 22

Säyfä Ar'ad, emperor 70, 72, 73

Saurma-Jeltsch, Lieselotte 166

Saxl, Fritz 141

Schapiro, Meyer 2, 12

Scrivener, Frederick Henry Ambrose 35

Sedulius 135, 140, 147

Sedulius Scottus 63

Sebastian, saint 233

Serenus of Marseilles 135

Servatius, saint 246

Sibylla, princess of Sweden 9

Simon, saint 10

Solomon 197, 202-206

Sophia, landgravine of Thuringia 233

Spingou, Fotini $X$

Squire, Michael 102

Stephen, martyr 225, 246

Step'anos Siwnec'i 102

Ström, Emilia 16

Swarzenski, Georg 2

Swithun, saint 225, 229
Täsfanä, Krəstos 72

Tatian of Adiabene VII, 12, 17, 21-26, 100, 140

-Diatessaron VII, 12, 13, 17, 18, 21, 22, 25, 26, 81, 100, 140, 174, 177

Terence $100,104,106-108,113,114$

Theon of Alexandria 101

Theophanes 34, 44

Theophanu, empress 205

Tilghman, Benjamin 166

Tisserant, Eugène, cardinal 9

Trinks, Stefan 133

Ulrich, saint 232

Underwood, Paul IX, 67, 75, 76, 81, 83, 88-90, 150, 151, 155

Van Gogh, Vincent 4

Van Gogh, Theo 4

Van Gogh, Vincent Willem 4

Van Rappard, Anton 4

Varro, Marcus Terentius 102

Vergil (Virgil) 100, 107, 108, 112, 122, 198, 201

Victor of Capua VII, 17, 21-26, 174

Vitus, saint 246

Vitruvius 198

von Euw, Anton 211

Wallraff, Martin 17, 30

Warin of Corvey, abbot 246

Watteau, Antoine 4

Watson, Francis X, 106, 110, 120-127

Weitzmann, Kurt 108, 113, 114, 120

Werner, Martin 87

Wibald of Stavelot, abbot 246

Wimmer, Hanna 47, 99, 133

Witakowska, Weronika 16

Wittekind, Susanne 133

Walker, Robert 147

Wormald, Francis 2, 135, 139, 140, 170

Zacchaeus 138, 141

Zachariah (Zacharias, Zechariah) 90, 138, 142

Zola, Nicholas J. 21

Zumthor, Paul 18

Zuurmond, Rochus 85, 92 